\title{
Expression of cytokeratin by malignant meningiomas: diagnostic pitfall of cytokeratin to separate malignant meningiomas from metastatic carcinoma
}

\author{
Yulin Liu, Charles D Sturgis, Mark Bunker, Reda S Saad, Ming Tung, Stephen S Raab and \\ Jan F Silverman
}

Department of Pathology and Laboratory Medicine, Allegheny General Hospital, Pittsburgh, PA, USA

\begin{abstract}
Based on clinical and histologic features, differentiating metastatic carcinomas from benign or malignant meningiomas usually is not difficult. Occasionally, however, in some patients without a clinical history of carcinoma, malignant meningiomas can morphologically simulate metastatic carcinoma, necessitating an immunohistochemical study for cytokeratin to make a correct diagnosis. However, the utility of immunohistochemical markers to separate malignant meningioma from metastatic carcinoma has not been investigated. The immunoperoxidase method with antigen retrieval was used to characterize the expression of three cytokeratins (AE1/AE3, CAM 5.2, and Pan cytokeratin), EMA, CEA, Ber-EP4, CD 15, and B72.3 in 12 previously diagnosed malignant meningiomas, 20 benign meningiomas, and 20 metastatic carcinomas. Cytokeratin expression was detected in $75 \%$ of malignant meningiomas, $0 \%$ of benign meningiomas, and $100 \%$ of metastatic carcinomas. While epithelial markers of Ber-EP4, CEA, B72.3 and CD-15 were positive in 90, 80, 70 and $65 \%$ of the metastatic carcinoma, respectively, they were negative in all 12 malignant meningioma examined. Vimentin immunoreactivity was seen in all benign and malignant meningiomas, and in $20 \%$ of metastatic carcinomas. Our results indicated that cytokeratin is not a reliable immunohistochemical marker to separate a malignant meningioma from metastatic carcinoma. A panel of epithelial markers including Ber-EP4, CEA, B72.3 and CD-15, and vimentin may be needed to separate malignant meningioma from metastatic carcinoma. Cytokeratin expression can be a potential pitfall for confusing a malignant meningioma with a metastatic carcinoma. Modern Pathology (2004) 17, 1129-1133, advance online publication, 7 May 2004; doi:10.1038/modpathol.3800162
\end{abstract}

Keywords: malignant meningioma; metastatic carcinoma; meningioma; cytokeratin; EMA; Ber-EP4; CEA; immunohistochemistry.

Meningioma is common intracranial neoplasm arising from the arachnoidal layer covering over the brain. Women are more commonly affected than men. Although most meningiomas grow slowly, they can occasional be difficult to remove due to brain involvement. Meningioma exhibits a histological diverse morphologic appearance with many subtypes described. ${ }^{1-5}$ In the new WHO brain tumor classification, meningiomas are categorized into three grades. ${ }^{2}$ While most grade I typical meningiomas are clinically benign, grade III malignant

Correspondence: Dr Y Liu, MD, PhD, Director of Cytology, Department of Pathology and Laboratory Medicine, Allegheny General Hospital, Drexel University School of Medicine, Pittsburgh, PA 15212, USA.

E-mail: yliu@wpahs.org

Received 18 August 2003; revised 24 February 2004; accepted 25 February 2004; published online 7 May 2004 meningiomas exhibit an aggressive clinical course including rapid growth, brain invasion, local recurrence, metastasis and poor prognosis. ${ }^{1,5-7}$ Histologically, malignant meningiomas are defined by morphologic features of frank malignancy far beyond the abnormalities present in atypical meningioma. These features include obvious malignant cytomorphology that resemble carcinoma, melanoma or sarcoma, or a high mitotic index with more than 20 mitoses per 10 high-power fields. ${ }^{2}$ Nuclear pleomorphism, necrosis, loss of the characteristic architecture of benign meningioma, increased cellularity and brain parenchyma invasion are usually present.

Since malignant meningioma can morphologically simulate metastatic carcinoma, separation of a malignant meningioma from metastatic carcinoma is very important due to markedly different treatment options and prognosis. Immunohistochemical 
study for cytokeratin has usually been applied to confirm or exclude a metastatic carcinoma. While keratin has been shown to be expressed in different subtypes of meningiomas, ${ }^{8-13}$ the diagnostic value of different cytokeratin expression in separating malignant meningioma from metastatic carcinoma have not been studied. To characterize the immunophenotypic features of the malignant meningiomas and to assess the diagnostic value of cytokeratin expression in separating malignant meningiomas from metastatic carcinomas, we studied the expression of cytokeratins, epithelial membrane antigen (EMA), vimentin and other epithelial antigens including carcinoembryonic antigen (CEA), Ber-Ep4, CD-15 and B72.3 by malignant meningiomas, benign meningiomas and metastatic carcinomas into the brain.

\section{Materials and methods}

Cases were retrieved from the archives of the Department of Pathology and Laboratory Medicine at Allegheny General Hospital from January 1986 to May 2003. A total of 52 brain neoplasms were studied. The original hematoxylin-eosin (H\&E)stained sections as well as a recut from each case were reviewed to confirm the diagnosis. All tumors were intracranial. The formalin-fixed paraffin-embedded blocks was selected from each case for immunohistochemical studies.

We studied 52 cases of brain neoplasms including 20 cases each of benign meningiomas and metastatic carcinomas, and 12 cases of malignant meningiomas. Cases of meningioma and malignant meningioma were classified according to the WHO classification of tumors of nervous system. ${ }^{2}$ Immunostaining was performed using the avidin-biotinperoxidase complex (ABC) method. Sections $5 \mu \mathrm{m}$ were cut from the paraffin blocks and mounted on charged slides. The sections were deparaffinized, rehydrated and treated with $1 \%$ hydrogen peroxide for $10 \mathrm{~min}$ to block endogenous peroxidase activity. Sections were then rinsed and incubated with primary antibodies cytokeratins (CK) (AE1/AE3, CAM 5.2, and wide spectrum keratin), EMA, CEA, Ber-EP4, CD 15, and B72.3. The immunostaining was performed on an automated immunostainer with appropriate positive and negative controls.

\section{Results}

The immunohistochemical findings are summarized in Table 1 and illustrated in Figures 1a-d. Cytoplasmic immunoreactivity of cytokeratin was seen in malignant meningiomas and metastatic carcinomas. For Ber-EP4, membrane rather than the cytoplasmic staining was counted as positive. For cytokeratins CAM 5.2, AE1/3 and Pan CK, all 20 metastatic carcinomas were strongly and diffusely positive. Nine cases $(9 / 12 ; 75 \%)$ of malignant meningiomas were positive for CAM 5.2, AE1/AE3 and Pan cytokeratin with strong and diffuse positivity in four cases $(33 \%)$ and focal positivity in five cases $(42 \%)$. No immunoreactivity was detected in any of the 20 cases of benign meningiomas.

EMA immunoreactivity was observed in benign (75\%, 15/20 cases) and malignant (75\%, 9/12 cases) meningiomas and metastatic adenocarcinoma $(80 \%$, $16 / 20$ cases). Strong and diffuse vimentin immunoreactivity was seen in all 12 malignant meningiomas and 20 benign meningiomas. A total of $20 \%$ metastatic carcinomas (5/20) were positive for vimentin with focal positivity seen in three cases and more diffuse staining in two cases.

For the epithelial markers, no immunoreactivity of Ber-EP4, CEA, CD-15 and B72.3 was observed in any of the 12 malignant meningiomas and 20 benign meningiomas. Metastatic carcinomas were positive for Ber-EP4, CEA, B72.3 and CD-15 in 90, 80, 70 and $65 \%$, respectively.

\section{Discussion}

Meningiomas are unique brain neoplasms with dual mesenchymal and epithelial differentiation. While most meningiomas display mesenchymal morphologic features such as fibrous, lipomatous and myxoid patterns, epithelial differentiation are demonstrated by ultrastructural evidence of desmosomes and histologic findings of papillary formation, clusters of epithelial-like cells, intracellular lumina, and immunoreactive for various

Table 1 Immunohistochemical findings

\begin{tabular}{|c|c|c|c|c|c|c|c|}
\hline Cases & $C K$ & $E M A$ & Vimentin & Ber-EP4 & $C E A$ & $B 72.3$ & $C D 15$ \\
\hline Malignant meningiomas (12 cases) & $\begin{array}{c}75 \% \\
(9 / 12)\end{array}$ & $\begin{array}{c}75 \% \\
(9 / 12)\end{array}$ & $\begin{array}{c}100 \% \\
(12 / 12)\end{array}$ & $\begin{array}{c}0 \% \\
(0 / 12)\end{array}$ & $\begin{array}{c}0 \% \\
(0 / 12)\end{array}$ & $\begin{array}{c}0 \% \\
(0 / 12)\end{array}$ & $\begin{array}{c}0 \% \\
(0 / 12)\end{array}$ \\
\hline Metastatic carcinoma (20 cases) & $\begin{array}{c}100 \% \\
(20 / 20)\end{array}$ & $\begin{array}{c}80 \% \\
(16 / 20)\end{array}$ & $\begin{array}{c}20 \% \\
(4 / 20)\end{array}$ & $\begin{array}{c}90 \% \\
(18 / 20)\end{array}$ & $\begin{array}{c}80 \% \\
(16 / 20)\end{array}$ & $\begin{array}{c}70 \% \\
(14 / 20)\end{array}$ & $\begin{array}{c}65 \% \\
(13 / 20)\end{array}$ \\
\hline Benign meningiomas (20 cases) & $\begin{array}{c}0 \% \\
(0 / 20)\end{array}$ & $\begin{array}{c}75 \% \\
(15 / 20)\end{array}$ & $\begin{array}{c}100 \% \\
(20 / 20)\end{array}$ & $\begin{array}{c}0 \% \\
(0 / 20)\end{array}$ & $\begin{array}{c}0 \% \\
(0 / 20)\end{array}$ & $\begin{array}{c}0 \% \\
(0 / 20)\end{array}$ & $\begin{array}{c}0 \% \\
(0 / 20)\end{array}$ \\
\hline
\end{tabular}



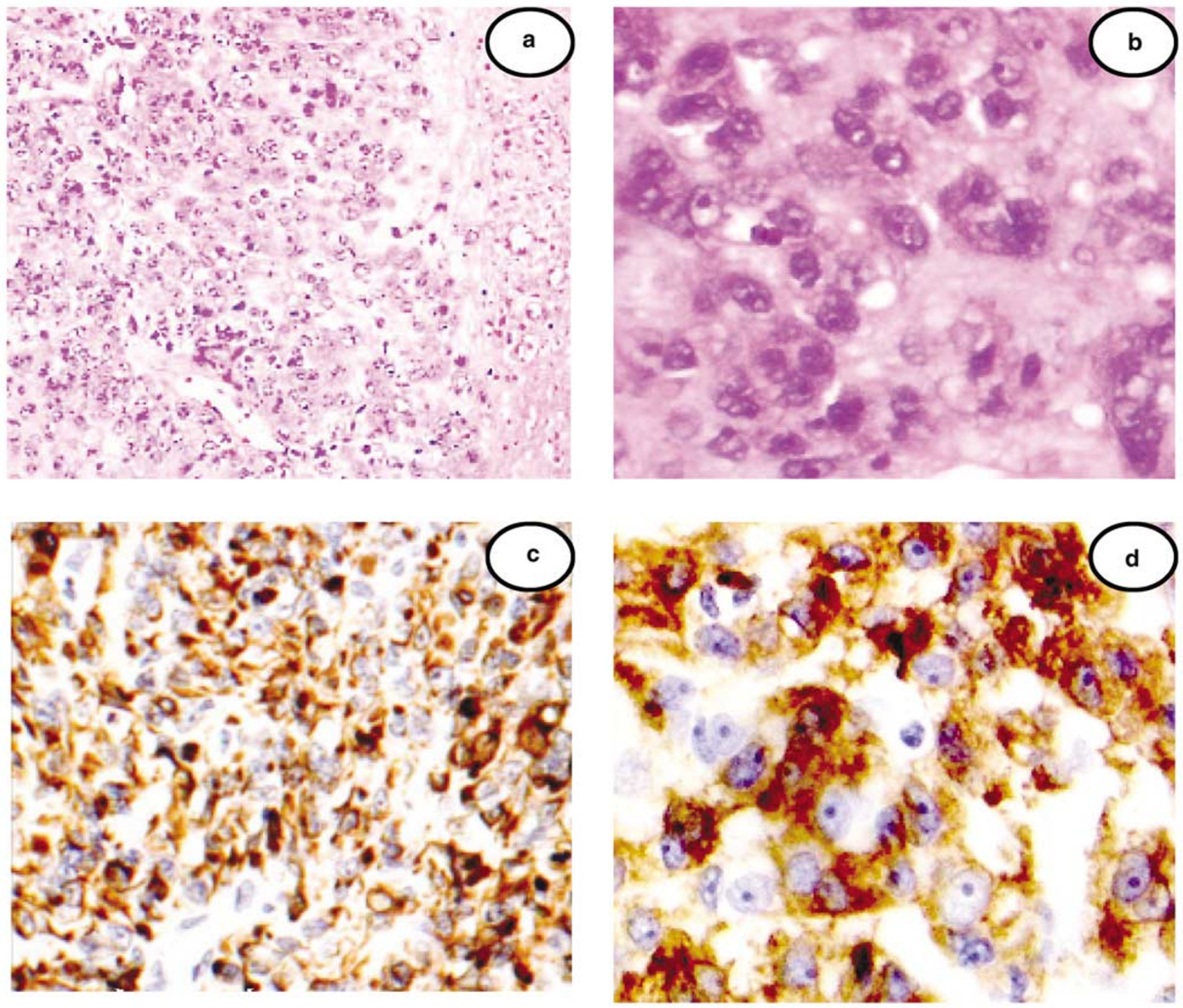

Figure 1 (a) and (b)-Malignant meningioma stained with H\&E. (a) $\times 300$; (b) $\times 600$. (c) and (d) —Diffuse and strong cytoplasmic immunoreactivity of cytokeratin in malignant meningioma. (c) $\times 300$; (d) $\times 600$.

epithelial markers. ${ }^{11,14-19}$ Although meningioma is a common neoplasm of the central nervous system, malignant meningioma is rare. The morphological features and the immunophenotype of benign meningioma have been extensively studied. Meningioma has been reported to be immunoreactive for EMA, vimentin and S-100. ${ }^{8,9,11,18,20-26}$ Secretory meningiomas have been shown to be immunoreactive for cytokeratin and usually behave in a more aggressive clinical course than other benign meningiomas. ${ }^{8,11-13,21,27}$

There are 12 malignant meningiomas evaluated in this study. While 9/12 cases of malignant expressed cytokeratin, none of the 20 benign meningioma was positive. Although, previous studies reported that the meningeal membrane as well as benign meningiomas can express some cytokeratin, ${ }^{9,22,25,28}$ we did not detect any cytokeratin immunoreactivity for
Cam 5.2, AE1/AE3 and Pan CK. The reason why malignant meningiomas can immunohistologically express cytokeratin is uncertain. Morphologically, 10 cases of malignant meningiomas showed areas of epithelial differentiation resembling carcinoma. Two cases had cells with pleomorphic/spindle configuration simulating metastatic sarcoma. While both malignant meningioma and metastatic carcinoma express EMA, none of the malignant meningioma was positive for markers often expressed by carcinoma such as CEA, Ber-EP4, B72.3 and CD-15. Among the epithelial markers that are positive for metastatic carcinoma, Ber-EP4 and CEA are the most useful ones with 90 and $80 \%$ sensitivity, respectively. Therefore, cytokeratin and EMA immunohistochemical studies cannot be utilized as markers to distinguish malignant meningioma from metastatic carcinoma. An immunohistochemical panel that 
includes cytokeratins, Ber-EP4, CEA, B72.3 and CD15 is needed to separate malignant meningioma from carcinomas, since all malignant meningiomas examined in this study were negative for CEA, BerEP4, B72.3 and CD-15.

Although vimentin can be expressed by some carcinomas, such as renal cell carcinomas, endometrial adenocarcinoma, sarcomatoid carcinoma, and some carcinomas of lung and breast origin, ${ }^{29-33}$ vimentin can be a useful marker to distinguish malignant meningioma from metastatic carcinoma. In our study, strong and diffuse vimentin immunoreactivity was seen in all 12 malignant meningiomas and 20 benign meningiomas, but only $20 \%$ (5/20 cases) of metastatic carcinomas with focal positivity seen in three cases $(12 \%)$. Therefore, negative vimentin immunoreactivity can help exclude either a benign or malignant meningioma.

Secretory meningioma is an uncommon histologic variant of meningioma characterized by epithelial differentiation with intracellular lumina containing PAS-positive eosinophilic material. Cytokeratin expression in secretory meningioma has been reported in a number of previous studies. ${ }^{11-13,27,34}$ Probsr-Cousin reported that 31 cases of secretory meningiomas expressed epithelial membrane antigen, cytokeratins, carcinoembryonic antigen, progesterone receptors and carbohydrate antigen 19-9. ${ }^{12}$ Assi et $a l^{11}$ studied the immunophenotype of nine cases of secretory meningioma with characteristic small gland-like lumina formation filled with a PAS-positive, diastase-resistant substance. All nine cases were positive for CK7, CK8, EMA and CEA, and negative for Ber-EP4, CD-15 and CK20. They concluded that the different expression of cytokeratin and negativity for Ber-EP4 and CD15 were useful for the distinction between secretory meningiomas and metastatic adenocarcinomas.

In summary, although cytokeratin expression does not separate a malignant meningioma from a metastatic carcinoma, a panel of epithelial markers including Ber-EP4, CEA, B72.3 and CD15 and vimentin may be useful in separating malignant meningioma from metastatic carcinoma. We believe that cytokeratin expression can be a potential pitfall for confusing a malignant meningioma with a metastatic carcinoma.

\section{References}

1 Niedermayer I, Kolles H, Henn W, et al. Meningioma. Classification and grading. Patholology 1997;18: 353-358.

2 Kleihues P, Louis DN, Scheithauer BW, et al. The WHO classification of tumors of the nervous system. J Neuropathol Exp Neurol 2002;6:215-225.

3 Fabiani A, Trebini F, Favero M, et al. The significance of atypical mitoses in malignant meningiomas. Acta Neuropathol 1977;38:229-231.
4 New PF, Hesselink JR, O’Carroll CP, et al. Malignant meningiomas: CT and histologic criteria, including a new CT sign. AJNR Am J Neuroradiol 1982;3:267-276.

5 Mahmood A, Caccamo DV, Tomecek FJ, et al. Atypical and malignant meningiomas: a clinicopathological review. Neurosurg 1993;33:955-963.

6 Coke CC, Corn BW, Werner-Wasik M, et al. Atypical and malignant meningiomas: an outcome report of seventeen cases. J Neurooncol 1998;39:65-70.

7 Nagashima G, Asai J, Suzuki R, et al. Different distribution of c-myc and MIB-1 positive cells in malignant meningiomas with reference to TGFs, PDGF, and PgR expression. Brain Tumor Pathol 2001;1:1-5.

8 Miettinen M, Paetau A. Mapping of the keratin polypeptides in meningiomas of different types: an immunohistochemical analysis of 463 cases. Hum Pathol 2002;3:590-598.

9 Ng HK, Tse CC, Lo ST. Meningiomas and arachnoid cells: an immunohistochemical study of epithelial markers. Pathology 1987;19:253-257.

$10 \mathrm{Ng} \mathrm{HK}$, Wong AT. Expression of epithelial and extracellular matrix protein markers in meningiomas. Histopathology 1993;22:113-125.

11 Assi A, Declich P, Iacobellis M, et al. Secretory meningioma, a rare meningioma subtype with characteristic glandular differentation: an histological and immunohistochemical study of 9 cases. Adv Clin Path 1999;3:47-53.

12 Probst-Cousin S, Villagran-Lillo R, Lahl R, et al. Secretory meningioma: clinical, histologic, and immunohistochemical findings in 31 cases. Cancer 1997;79:2003-2015.

13 Colakoglu N, Demirtas E, Oktar N, et al. Secretory meningiomas. J Neurooncol 2003;62:233-241.

14 Carlotti Jr CG, Neder L, Colli BO, et al. Clear cell meningioma of the fourth ventricle. Am J Surg Pathol 2003;27:131-135.

15 Hill JR, Kuriyama N, Kuriyama H, et al. Molecular genetics of brain tumors. Arch Neurol 1999;56: 439-441.

16 McLean CA, Jolley D, Cukier E, et al. Atypical and malignant meningiomas: importance of micronecrosis as a prognostic indicator. Histopathology 1993;23: 349-353.

17 Yates AJ. An overview of principles for classifying brain tumors. Mol Chem Neuropathol 1992;17: 103-120.

18 Ang LC, Taylor AR, Bergin D, et al. An immunohistochemical study of papillary tumors in the central nervous system. Cancer 1990;65:2712-2719.

19 Jellinger K, Slowik F. Histological subtypes and prognostic problems in meningiomas. J Neurol 1975; 208:279-298.

20 Al-Sarraj S, King A, Martin AJ, et al. Ultrastructural examination is essential for diagnosis of papillary meningioma. Histopathology 2001;38:318-324.

21 Alguacil-Garcia A, Pettigrew NM, Sima AA. Secretory meningioma. A distinct subtype of meningioma. Am J Surg Pathol 1986;10:102-111.

22 Barnhart KF, Wojcieszyn J, Storts RW. Immunohistochemical staining patterns of canine meningiomas and correlation with published immunophenotypes. Vet Pathol 2002;39:311-321.

23 Giuffre G, Vitarelli E, Tuccari G, et al. Immunohistochemical study of Tn, sialosyl-Tn and T antigens in human meningiomas. Eur J Histochem 1998;42: 197-203. 
24 Kasantikul V, Punyavoravut V, Maneesri S, et al. Mucin-producing malignant meningioma with papillary and glandular patterns. J Med Assoc Thai 1995;78: 635-640.

25 Radley MG, di Sant'Agnese PA, Eskin TA, et al. Epithelial differentiation in meningiomas. An immunohistochemical, histochemical, and ultrastructural study - with review of the literature. Am J Clin Pathol 1989;92:266-272.

26 Terpe HJ, Kasper M, Martin H, et al. Detection of cytokeratin in cells of the arachnoid and in meningiomas. Zentralbl Allg Pathol 1988;134:259-264.

27 Slowinski J, Kaluza J, Sabat D, et al. Secretory meningioma of the brain. Report of two cases. Folia Neuropathol 1999;37:87-92.

28 Artlich A, Schmidt D. Immunohistochemical profile of meningiomas and their histological subtypes. Hum Pathol 1990;21:843-849.

29 Dabbs DJ, Geisinger KR, Norris HT. Intermediate filaments in endometrial and endocervical carcinomas. The diagnostic utility of vimentin patterns. Am J Surg Pathol 1986;10:568-576.
30 Waldherr R, Schwechheimer K. Co-expression of cytokeratin and vimentin intermediate-sized filaments in renal cell carcinomas. Comparative study of the intermediate-sized filament distribution in renal cell carcinomas and normal human kidney. Virchows Arch A Pathol Anat Histopathol 1985; 408:15-27.

31 Santini D, Bazzocchi F, Paladini G, et al. Intermediatesized filament proteins (keratin, vimentin, desmin) in metaplastic carcinomas, carcinosarcomas and stromal sarcomas of the breast. Int J Biol Markers 1987;2:83-86.

32 Cappello F, Aragona F, Serretta V, et al. Sarcomatoid carcinoma of urinary bladder: immunohistochemical study of an uncommon case. Urol Int 2002;69: 141-144.

33 DeLong W, Grignon DJ, Eberwein P, et al. Sarcomatoid renal cell carcinoma. An immunohistochemical study of 18 cases. Arch Pathol Lab Med 1993;117:636-640.

34 Stavale JN, Malheiros SM, Gabbai AA. Secretory meningioma. Report of two cases. Arq Neuropsiquiatr 1997;55:478-481. 